\title{
Reflujo Gastroesofágico: Evolución Clínica y Radiológica
}

\author{
Dra. Maureen Rossel G. ${ }^{1}$, Dr. Sergio Ceresa $0^{2}{ }^{2}$, Dr. Julio Espinoza M. ${ }^{3}$ \\ Gastro-esophageal Reflux: Clinical and Radiological evolution
}

\begin{abstract}
Ninety children with clinical and radiological gastroesophageal reflux were evaluated. Ages at first consultation were: less than 12 months: 63 ; $12-24$ months: 18 ; and older than 24 months: 10 .

Vomiting $(71.1 \%$ and respiratory symptoms $(24.5 \%)$ were the most frequent causes of consultaton. Medical treatment (upright position above 45\%; thickened, small, frequent feedings and methoclopramide $(0.3 \mathrm{mg} / \mathrm{kg} / 24 \mathrm{hrs})$ was followed by clinical improvement in 81 patients $(64.2 \%$ of them in less than 6 months). 9 subjects requìred surgical treatment.

The medical treatment was strictly followed, irregularly followed or not accepted in $62.2 \%$; $26.7 \%$ and $11.1 \%$ respectively. Control esophagogram 8 to 12 months after the beginning of treatment were normal in 40 children. Twenty four children showed persistence of gastroesophageal reflux. In the 81 patients clinically recovered, weight/height rates under 25 percentile were present in $32 \%$, at the beginning, and in $18.6 \%$ at the end of the study, respectively.
\end{abstract}

El Reflujo Gastroesofágico (RGE) es un trastorno de la función del esófago distal que facilita el retorno del contenido gástrico hacia el esófago? Se acompaña frecuentemente de vómitos y/o regurgitaciones. Los sintomas se presentan en el primer trimestre de la vida en $98 \%$ de los pacientes.

En la evolución natural de esta afección se ha observado que aproximadamente $60-65 \%$ de los pacientes estan sin síntomas a los 2 años de edad, y un 30\% continúa con molestias más allá de los 4 affos. La estenosis esofágica se encuentra en $5 \%$ de los enfermos y un porcentaje similar muere en ausencia de ésta ${ }^{2}$.

El RGE se relaciona con otros problemas médicos: síndrome de muerte súbita ${ }^{3 \cdot 4}$; enfermedades broncopulmonares ${ }^{5-8}$ (síndrome bronquial obstructivo recidivante (S.B.O.R.), neumopatía aspirativa, neumonitis); síndromes neuropsiquiátri$\cos ^{9}$ (retraso del desarrollo psicomotor, irritabilidad, disfagia psicógena); y retraso pondoestatu . $\mathrm{ral}^{2-10-12}$.

El tratamiento del RGE es médico (posición levantada, alimentos espesos y probablemente metoclopramida) $y$, si éste fracasa, quirúrgi$\mathrm{co}^{1-3-7-10-13}$. En nuestro país existen pocos estudios que permiten evaluar el tratamiento médico.

El objetivo de este trabajo, en consecuencia, fue analizar. la evolución clínica y radiológica de niños

1 Universidad de Chile, Facultad de Medicina, División de Ciencias Médicas Sur, Depto. Pediatría y Cirugía Infantil (Central). Trabajo de incorporación a la Sociedad Chilena de Pediatría. Jornadas de Pediatría, Lo Basnechea, Diciembre 1981

2 Unidad de Gastroenterología Pediátrica, Servicio de Pediatría, Hospital Paula Jaraquemada.

3 Universidad de Chile, Instituto de Nutrición y Tecnología de los Alimentos (INTA). controlados en el policlinico de Gastroenterología Infantil con el diagnóstico de Reflujo Gastroesofágico.

\section{SUJETOS Y PROCEDIMIENTOS}

Se estudiaron 90 nifios controlados en el policlínico de Gastroenterología Infantil entre 1977 y 1981 , con el diagnóstico de RGE.

El diagnóstico se basó en criterios clínicos ${ }^{1}$ y fue confirmado radiologicamente por el Tránsito Esófago-Estómago y Duodeno (TEED). La evolución clinica se controló mensualmente y la radioló. gica cada 8 a 12 meses dependiendo de los síntomas. Se evaluaron los siguientes parámetros de las manifestaciones de RGE: síntomas de consulta; edad de presentaciốn de los síntomas y de la primera consulta; rechazo o aceptación del tratamiento médico, evolución de los síntomas y respuesta al tratamiento médico; evolución radiológica; indjcaciones de intervención quirúrgica; $y$, relación del RGE con el estado nutritivo.

El tratamiento médico indicado fue: posición levantada sobre $45^{\circ}$, alimentos espesos, fraccionados y metoclopramida $0.3 \cdot 0.5 \mathrm{mg} / \mathrm{kg} / \mathrm{d}$ ía.

Los niños fueron controlados cada 15 a 30 días para evaluar el tratamiento. Se calificó al tratamiento médico en: a) seguido estrictamente, cuando la madre cumplia todas las indicaciones correctamente; b) seguido irregularmente, cuando la madrè cumplía bien las indicaciones en algunos periodos de tiempo o sólo algunos aspectos del tratamiento; c) rechazado, cuando la madre no cumpl ja las indicaciones.

Los pacientes fueron agrupados en aquellos en que los sintomas habian desaparecido en un periodo menor de seis meses, y en los que la mejoría ocurrió entre seis meses y un año. Se consideró 
mala evolución clínica y fueron intervenidos quirúrgicamente, aquellos en que la intensidad y/o frecuencia de aparición de los síntomas no mejoró o en que hubo complicaciones derivadas de su patologia.

El estado nutritivo se analizó según la curva de relación peso/talla del National Center for Health Statistics (NCHS), cuyos valores normales se encuentran entre los percentiles 25 y $75^{14.15}$.

\section{RESULTADOS}

Los sintomas de consulta fueron: vómitos y regurgitaciones (habitualmente postprandiales, 20 más veces al día y de volumen variable), en $71.1 \%$ de los casos; síntomas broncopulmonares (tos, difjcultad respiratoria, disnea, espiración prolongada, presencia de estertores húmedos y sibilancias), en $24.5 \%$ (sindrome bronquial obstructivo recidivante $20 \%$ y neumopatía aspirativa $4.5 \%$ ); crisis de cianosis $2.2 \%$; disfagia $1.1 \%$ y anorexia $1.1 \%$. A ellos se agregaron otros como pirosis, disfagia, dolor cólico abdominal, hemorragia digestiva $y$ anemia.

La edad de comienżo de los sintomas fue antes de $\operatorname{los} 3$ meses y entre 3 y 6 meses, en $80 \%$ y $92.2 \%$ de los enfermos respectivamente. El $6.7 \%$ inició los síntomas entre 6 y 12 meses y el $1.1 \%$ en edades posteriores.

La edad de la primera consulta en el policlínico de Gastroenterologia fue: menores de 6 meses $37.8 \%$; 6 y 12 meses $31.1 \% ; 12$ y 18 meses $13.3 \%$; y 18 y 24 meses $6.7 \%$; y sobre 24 meses $11.1 \%$.

El tratamiento fue seguido estrictamente en el $62.2 \%$ de los casos, irregularmente en $26.7 \%$ y rechazado en e] $11.1 \%$. El aspecto menos aceptado fue la posición.

La evolución clínica fue buena en $90 \%$ de los niños $(64.2 \%$ de ellos mejoró en un periodo menor de 6 meses y los restantes en un período mayor de 6 meses). En 9 enfermos (10\%) la mala evolución cl jínica determinó la indicación quirúrgica.

Tabla 1.

Respuesta de los síntomas de Relujo Gastroesofágico al tratamiento médico. $\mathrm{N}=90$

B $\% \quad$ M $\%$

Vómitos y

$\begin{array}{llllll}\text { Regurgitaciones } & 64 & 58 & (90.6) & 6 & (9.4)\end{array}$

Síntomas

Respiratorios

2416

(66.7) 8

Disfagia

11

Anorexia

11

*EL TEED de control fue normal en 3 de estos enfermos y continúan en control en Policiínico Buoncopulmonares.
La respuesta de los sintomas de RGE al tratamiento médico se analiza en la Tabla 1 . Del total de 90 pacientes, 64 consultaron por sintomas gas. trointestinales, que en su mayoría $(90.6 \%)$ mejoró con el tratamiento médico. 24 enfermos consultaron por síntomas broncopuimonares, de los cuales 66.7\% tuvo buena evolución.

En todos los enfermos se hizo por lo menos un TEED. En 26 enfermos no se tomó un segundo tránsito porque abandonaron sus controles siendo clínicamente asintomáticos.

\section{Tabla 2.}

Respuesta de los síntomas en 64 enfermos en tratamiento por Reflujo Gastroesofágico y con más de un TEED*

Respues ta de los síntomas al tratamiento

$\begin{array}{lccccc}\text { Síntomas } & \mathrm{N}^{+} & \text {Buena } & (\%) & \text { Mala } & \text { (\%) } \\ \text { Digestivos } & 58 & 53 & (91.4) & 5 & (8.6) \\ \text { Broncopulmonares } & 37 & 28 & (75.7) & 9 & (24.3)\end{array}$

*TEED = Tı́́nsito Esófago-Estómago y Duodeno.

${ }{ }_{N}=$ frecuencia de aparición del sintoma en los 64 enfermos.

En 64 enfermos, el TEED fue repetido $y$ se demastró normal en 40 (62.5\%) (Tabla 2). Los sintomas digestivos mejoraron en $91.4 \%$ de los 64 . Los sintomas broncopulmonares tuvieron buena respuesta en $75.7 \%$.

Tabla 3.

Respucsta de los sintomas en 40 enfermos en tratamiento por Reflujo Gastroesofágico y con TEED* de control normal

Sintomas
Digestivos
Mixcos
Broncopulmonares
"TEED: Tránsito Esófago-Estómago y Duodeno.
Enfermos en control por Policlínico Broncopulmonares.

La Tabla 3 muestra la respuesta de los síntomas en los 40 enfermos en que el TEED de control fue normal: Los síntomas digestivos mejoraron en todos ellos. En cambio, los síntomas broncopulmonares tuvieron una buena evolución clinica y radiológica en $86.4 \%$. Los 3 enfermos (13.2\%) con mala evolución clínica, pero buena evolución radiológica, continúan en control en el policlínico de Broncopulmonares. 
En la Tabla 4 se observa la respuesta de los sintomas en 24 enfermos con más de 1 TEED, en que el último estudio radiológico continuaba positivo para RGE: Los sintomas digestivos respondieron bien en $73.9 \%$, y persistieron en $26.1 \%$. Los sintomas de respiratorios tuvieron buena respuesta clínica en $66.7 \%$, y mala respuesta, clínica y radiológica en $33.3 \%$.

\section{Tabla 4.}

Respuesta de los sintomas en 24 enfermos en tratamiento por Reflujo Gastroesofágico y con TEED* de control positivo para Reflujo Gastroesofágico.

Respuesta de los síntomas al trataraiento

\section{Síntomas}

Buen

(\%)

Mala

(\%)

Mixtos

(73.9)

6

(26.1)

(66.7)

5

(33.3)

*TEED: Tránsito Esófago-Estómago y Duadeno.

Las indicaciones de intervención quirúrgica ( 9 de 90 niffos) fueron: hemorragia digestiva, vómitos persistentes, neumopatía aspirativa, síndrome bronquial obstructivo recidivante (descartadas otras causas broncopulmonares), incapacidad materna de cumplir el tratamiento (coeficiente intelectual bajo).

En los 81 niños con RGE que evolucionaron bien con el tratamiento médico, la relación peso/talla estuvo bajo el percentil 25 en $32 \%$ al ingreso $y$ en $18.6 \%$ al término de la experiencia. En cambio de los 9 niños que necesitaron tratamiento quirúrgico, el $44.4 \%$ estaba bajo el percentil 25 , proporción que no se modificó con el tratamiento médico. El parámetro peso/talla fue una de las variables consideradas para la intervención quirúrgica.

\section{COMENTARIO}

Los resultados obtenidos en el presente trabajo son concordantes con lo publicado previamente, ya que confirman el hecho de que los sintomas de RGE aparecen precozmente en el lactante ${ }^{2}(92.2 \%$ antes de los 6 meses). La primera consulta, en cambio, se realiza dentro de los primeros 2 años de vida.

E] síntoma de presentación más frecuente en nuestra experiencia fue el vómito $(71.1 \%$ de los niños), mientras que para Herbst ${ }^{1}$, este constituye el $90 \%$. Cuando el vómito es muy frecuente puede llevar a retraso pondoestatural ${ }^{10-13}$, lo que observamos en el 33.3\% de los niños de nuestro estudio.

El RGE también se asocja a patología bronco- pulmonar $^{5-6-7}$. En este estudio observamos que $24.5 \%$ de los niños consultó por síntomas respiratorios, representados en su mayor parte por sindrome bronquial obstructivo recidivante (S.B.O.R.).

Una historia clínica sugerente, junto a un TEED que lo dernuestra, es adecuado en la mayoria de los casos para el diagnóstico de RGE. Los otros test diagnósticos ( $\mathrm{pH}$, esofagoscopía, biopsia, manometría de esófago, cintigrafía, etc.) son necesarios cuando hay discordancia entre la historia clínica y TEED, o clando se busca complicaciones del RGE $1 \cdot 13 \cdot 16 \cdot 17$.

Cuando la historia clínica y el TEED demuestran la presencia de RGE, debe indicarse tratamiento médico riguroso ${ }^{1-13}$, para lo cual es necesario educar a los padres informándoles del problema del nifo y los principios en que se basa el tratamiento. Hay algunos aspectos que deben adaptarse a cada situación clínica, por ejemplo, la posición debe ser más cercana a los $90^{\circ}$ de inclinación si el paciente no responde con una inclinación inicial de por to menos $45^{\circ}$. Cuando hay sintomas respiratorios seria más recomendable la posición prono en $30^{\circ}$ de inclinación, ya que disminuye la posibilidad de aspiración ${ }^{1-18}$. Esta técnica no fue usada por nosotros.

La eficacia de la alimentación con pequeños volúmenes $y$ fraccionada ha sido corroborada por los radiólogos al observar que la ingestión de cantidades grandes de Bario permitè la aparición de RGE aún en aquellos niños en que con técnicas adecuadas, estandar, no la tienen ${ }^{1}$. El tratamiento médico no produce resultados inmediatos $y$, puede haber un lapso de 2 semanas o más antes de que se evidencie mejoria clínica. Generalmente debe existir 4 a 6 semanas de tratamiento médico bjen llevado para poder evaluarlo ${ }^{1-19}$. En nuestra serie obseryamos que el tratamiento médico fue seguido estrictamente en $62.2 \%$ de los niños, siendo el aspecto más rechazado la posición.

Los síntomas gastrointestinales responden mejoc al tratamiento médico que los broncopulmonares $(90.6 \%$ y $66.7 \%$ de buena respuesta respectivamente).

En los pacientes con TEED de control negativo para RGE, los sintomas gastrointestinales habian mejorado en el $100 \%$, en cambio, los síntomas broncopulmonares en $86.4 \%$. El resto (13.2\%) continúa en el policlínico de broncopulmonares. Esto apoyaria la idea de que el hallazgo de enfermedad de las vias respiratorias y RGE no necesariamente significa una relación causal $y$ exige el estudio de otras posibilidades etiológicas para el problema broncopulmonar ${ }^{1}$. La correlación de sintomas clínicos y signos radiológicos es adecuada en la mayoría de los enfermos. La mejoría clínica 
precedió a la rađiológica en algunos (Tabla 4). Estos pacięntes, en que sólo el TEED persiste positivo para RGE, continúan en tratamiento médico.

La evolución clínica de nuestros enfermos es similar a la descrita en la titeratura, ya que en $90 \%$ de los niños fue buena con el tratamiento médico. La mala evolución en el $10 \%$ de los pacientes estudiados determinó la indicación quirúrgica. El tratamiento médico es todavia más confiable en el niño menor donde la historia natural sugiere buen pronósticol-20.

La cirugía como tratamiento del RGE, debiera emplearse en una minoría de pacientes con severas complicaciones, o que continúan con síntomas significativos, a pesar de un tratamiento médico bien llevado ${ }^{1 \cdot 13}$. La cirugía tiene indicación inmediata cuando hay condjciones que ponen en peligro la vida, como neumopatía por aspiración, apnea recurrente, etc. ${ }^{13}$. Si no existen estas condiciones debe darse un plazo de por lo menos 6 semanas de tratamiento médico adecuado ${ }^{13-19}$. De los 9 niños que necesitaron tratamiento quirúrgico, 3 fueron operados precozmente, debido a hemorragia digestiva, en 2 casos, y neumopatia aspirativa en 1 paciente. Debe considerarse también aquellos factores familiares o ambientales que impiden un tratamiento médico adecuado, con persistencia de los síntomas, como lo observamos en uno de los niños en que el tratamiento médico fue repetidamente rechazado por la madre.

El diagnóstico de reflujo gastroesofágico en la práctica diaria de policlínico puede basarse en la clínica y radiología. Una minoría requiere exáme. nes más complejos. El tratamiento médico resulta en la mejoría cínica y radiológica de la mayoría de los enfermos, especjalmente si asisten regularmente a control y siguen las indicaciones estrictamente. El médico debe estar alerta a las complicaciones de la enfermedad y $/ 0$ a la persistencia de sus síntomas pues el fracaso del tratamiento médico conduce a considerar el quirúrgico.

En resumen, los síntomas clínicos de Reflujo Gastroesofágico aparecen precozmente en el lactante, sin embargo, la consulta médica se realiza meses después. Los síntomas más frecuentes de presentación son de origen gastrointestinal o broncopulmonares. El tratamiento de elección del RGE fue el tratamiento médico y su fracaso determinó la indicación quirúrgica. El sintoma vómito $y / o$ regurgitación tiene mejor respuesta al tratamiento médico que los síntomas broncopulmonares. La mejoría clínica se correlacionó bien con la mejoría radiológica.

\section{RESUMEN}

Se evaluaron 90 niffos con RGE clínico y radiológico, cuyas edades en la primera consulta fueron: menos de 12 meses: $62 ; 12.24$ meses; 18 ; y mayor de 24 meses: 10.

Consultaron por vómitos el $71.1 \%$ y por sintomas broncopulmonares $24.5 \%$. Con el tratamiento médico (posición levantada $45^{\circ}$, dieta espesa y fraccionada y metoclopramida $0.3 \mathrm{mg} / \mathrm{kg} / \mathrm{dia}$ ) mejoraron clínicamente 81 enfermos $(64.2 \%$ de elfos en menos de 6 meses). 9 fueron intervenidos quirurgicamente.

El tratamiento médico fue seguido estrictamente, irregularmente o rechazado en $62.2 \% ; 26.7 \%$ y $11.1 \%$ respectivamente. El esofagograma de control 8-12 meses despues de iniciado el tratamiento fue normal en 40 niños y demostró RGE en. 24 niños. De los 81 recuperadōs clínicamente la rela. ción peso/talla al ingreso y al término de la experiencia fue bajo el 25 percentil en $32 \%$ y $18.6 \%$ respectivamente. 26 niños abandonaron los controles, siendo asintomáticos.

\section{REFERENCIAS}

${ }^{1}$ Herbst, J.J. Gastroesophageal Reflux. J. Pediatr. 98: $859,1981$.

${ }^{2}$ Carre, I.J. The natural history of the partial thoracic stomach ("Hiatal hernia") in children. Arch. Dis. Child. 34 : 344, 1959.

${ }^{3}$ Herbst, J.J, Book, L.S. and Bray, P.F. Gastroesophageal reflux in the "Near Miss" sudden infant death syndrome. J. Pediatr. 92: 73, 1978 .

4 Leape, L.L., Holder, T.M., Fraklin, J.D., Amoury. $R . A$, Ascraft, K.W. Respiratory arrest in infants secondary to Gastroesophageal reflux. Pediatrics 60: $924,1977$.

5 Danus, O., Casar, C. Larrain, A. and Pope, C.E. II. Esophageal reflux an unrecognized cause of recurrent obstructive bronchitis in children. J. Pediatr. 89 : 220, 1976.

${ }^{6}$ Euler, A.R., Byrne, W.J., Ament, M.E., Fonkalstud, E.W., Strobel, C.T., Siegel, S.C., Katz, R.M. and Rachelefsky, G.S. Recutrent pulmonary disease in children: A complication of gastroesophageal reflux. Pediatrics 63: 47, 1979.

${ }^{7}$ Darling, D.B., McCauley, R.G.X., Leonidas, J.C. Schwartz, A.M. Gastroesophageal reflux in infants and children: Correlation of radiological severity and pulmonary pathology. Radialogy 127: 735, 1978.

${ }^{8}$ Espinoze, J., Heitman, P. The gastroesophageal sphincter in the first year of life. Gastroenterology 4: 773,1971

${ }^{9}$ Bray, P.F., Herbst, J.J., Johnson, D.G., Book, L.S., Ziter, F.A., London, V.R. Childhood gastroesophageal reflux: Neurologic and psychiatric syndromes mimicked. JAMA 237: 1342, 1977.

10 Kibel, Maurece A. Gastroesophageal reflux and failure to thrive in infancy: Proceeding of the $76 \mathrm{th}$. Ross Conference on Pediatric Research, 1979, Pag. 39.

11 Carré, IJ., Anderson, C.M., Burke, V. (eds) Pediatric Gastroenterology. Oxford UK, Blackwell Scientific Publications, 1975.

12 Euler, A.R., Ameni, M.E. Gastroesophageal reflux in children. Ann. Pediat. 22: 43, 1976.

13 Leape, $L$, and Ramenofsky, $M$. Surgical treatment of gastroesophageal reflux in children. Am. J. Dis. Child. 134: $935,1980$. 
14 Hamill, P. \& Drizd, $T$. Physical growth: National Center for Health Statistics Percentiles. Am. J. Clin. Nutr. 32: 607, 1979.

is U.S. Department of Health. N.C.H.S. Growth Charts. Monthly Vital Statistics Report. Vol. 25, No 3, 1976.

16 Arasu, T.S. Gastroesophageal reflux in infants and children: Comparative accuracy of diagnostic methods. J. Pediatr. 96: 798, 1980.

17 Moroz, S.P., Espinoza, J., Cumming, W.A., Dirmant, N.E. Lower esophageal sphincter function in children with and without gastroesophageal reftux. Gastroenterology $71: 236,1976$.
${ }^{3}$ Meyer, W.F., Herbst, J.J. and Jolley, S.G. Superior efficacy of the prone elevated position in treatment of post-prandial gastroesophageal reflux. Ped. Res. 15: $541,1981$.

19 Carre, \&. Postural treatment of infants and young children with a Hiatal Hemia (Partial Thoraxic Stomach). Proceedings of the 76 th. Ross Conference on Pediatric Research, 1979. Pag. 86.

20 Leape, L. Gastroesophageal reflux, Proceedings of the 76 th. Ross Conference on Pediatric Research, Pág. 75. 1979. 